\title{
Utility of perioperative B-type natriuretic peptide in off-pump bypass surgery
}

\author{
Dikshya Joshi, Zhi Gang Guo \\ Department of Cardiothoracic Surgery, Tianjin Chest Hospital, Tianjin 300350, P.R. China
}

\section{Citation}

Dikshya Joshi, Zhi Gang Guo. Utility of perioperative B-type natriuretic peptide in off-pump bypass surgery. Nepalese Heart Journal 2014;11(1): 19-25.

\section{Keywords:}

B-type natriuretic Peptide, bypass surgery, mechanical ventilation, NYHA, OPCAB

\section{INTRODUCTION}

BNP is a cardiac neurohormone released by ventricular myocytes in response to ventricular dysfunction and wall stress. ${ }^{1}$ Its primary function is to defend against volume overload; the specific actions include natriuresis, inhibition of the renin-angiotensin-aldosterone axis and inhibition of sympathetic nerve activity. ${ }^{2} \mathrm{BNP}$ is regarded as an important

\section{ABSTRACT}

Background and Aims: This study was performed to determine clinical relevance of perioperative B-type natriuretic peptide $(B N P)$ in patients undergoing off-pump coronary artery bypass grafting.

Methods: 145 consecutive patients undergoing off-pump coronary artery bypass grafting during 8-month period were enrolled in this study. The relationship between the plasma BNP and various clinical parameters was examined. Postoperatively their main clinical endpoints including requirement of mechanical ventilator support, length of intensive care unit stay and hospital stay was closely monitored.

Results: Mean preoperative BNP levels were significantly higher in patients whose left ventricular ejection fraction was less than $0.50(P<0.00083)$, and New York Heart Association class (III, IV) $(P<0.02)$. The determinants of preoperative higher level of $B N P$ can be related to the advanced age of the patients, $r=0.387$ $(P<0.01)$ and left ventricular end diastolic diameter, $r=0.200$ $(P<0.05)$. Postoperative 12-hour BNP correlated significantly with the duration of mechanical ventilation, rho $=0.84(P<0.05)$, and postoperative hospital stay for 10 days or more, rho=0.202 $(P<0.05)$. Logistic regression analyses showed a significant association between 12-hour BNP and the requirement of mechanical ventilation, Wald $=3.956(P<0.049$, 95\% CI_1.02320.476).

Conclusion: Plasma BNP concentration is a valuable biochemical marker, is easy to measure and can effectively predict postoperative outcome in off-pump coronary artery bypass grafting. Baseline $B N P$ had strong correlation with the age and ventricular function of the patient. Postoperatively, elevated 12-hour BNP indicated prolonged ventilation and longer duration of hospital stay.

\section{Corresponding author}

Zhi Gang Guo

Department of Cardiothoracic Surgery, Tianjin Chest Hospital, Tianjin 300350, P.R. China

Email:xkguozhigang@gmail.com 
parameter to aid diagnosis, assess prognosis, and conduct risk stratification in various heart diseases, and hence represents one of the most important diagnostic and therapeutic substances to be introduced in the last decade. , $3,4,5^{2}$

Off-pump coronary artery bypass (OPCAB) grafting via median sternotomy is a revolutionary procedure that results in fewer postoperative complications and less myocardial injury. ${ }^{5}$ Despite the advances in operative techniques, OPCAB is still accompanied with the inflammatory response caused by surgical trauma or reperfusion injury, and is associated with significant risk for postoperative cardiac events. ${ }^{6,7}$ The predictive value of BNP in cardiac surgeries including OPCAB has shown some promising results. ${ }^{3}$ Elevated BNP levels can provide valuable information in the risk stratification of the patient, and may also help in disposition decisions.

Present study is focused to elaborate the changes in BNP levels at different times during the perioperative period in patients undergoing OPCAB surgery in order to correlate the data with interim clinical status of the patient.

\section{METHODS}

This prospective observational cohort study was conducted in cardiac surgery department, of Tianjin Chest Hospital, Tianjin, China. The data was collected within eight-month period from October 252010 to June 10 2011. One hundred and forty five consecutive patients undergoing OPCAB were included in this study. Blood samples for BNP measurement were obtained within 24 hours before surgery and serially at 6 hours, 12 hours, 24 hours, and 7 days after the surgery. BNP was measured using the Triage B-type natriuretic peptide test (Biosite Incorporated, San Diego, USA).Blood samples were collected into Ethylene diaminetetra-acetic acid (EDTA) coated tubes containing $500 \mathrm{IU} /$ $\mathrm{ml}$ aprotinin. BNP concentration was determined by a competitive enzyme-linked immunosorbent assay (ELISA) in 96-well microtiter plates (micro-ELISA) ${ }^{8}$ The test was approved by the local ethical committee. Informed consent was obtained from all patients. Patients were excluded from the study if they had severe renal dysfunction or liver failure and/or pre-existing autoimmune diseases.

Standardized anesthesia was used in all patients, and OPCAB was undertaken through a median sternotomy. Postoperative treatment in Intensive care unit (ICU) was standardized. Ventilation time, ICU stay time and hospital time were followed.
All data were analyzed using the statistical package SPSS (version 19.0; SPSS Inc., Chicago, IL). Continuous data are presented as mean \pm standard deviation and categorical data as absolute numbers and percentages. Because BNP values were not normally distributed, they were logarithmically transformed for statistical analysis, but are presented in the study as non-transformed median and range. Correlations between continuous variables were assessed using Pearson's correlation. The Student's t-test and chi-square test were used for comparisons of continuous and categorical variables between groups respectively. The difference in variables was evaluated by the Analysis of variance (ANOVA) test. The time course of BNP and cardiac Troponin within each group was tested by ANOVA for repeated measures followed by a Bonferroni post-hoc test. The groups of preoperative BNP level were dichotonized to being less than $100 \mathrm{pg} / \mathrm{ml}$ or more than $100 \mathrm{pg} / \mathrm{ml}$. A linear regression model was used to assess the association between the log-transformed 12hour BNP levels and the primary predictor. The predictive value of BNP concentrations and the clinical outcome was assessed by use of logistic regression. The utility of BNP as a prognostic indicator of postoperative complications was evaluated using receiver operating characteristic (ROC) curves. $\mathrm{P}$ values $<0.05$ were considered to be statistically significant.

\section{RESULTS}

All patients completed the test and there were no deaths. However, three patients developed serious postoperative complications. One patient required placement of an intraaortic balloon pump for refractory low cardiac output. One patient developed ventricular fibrillation and had to be defibrillated postoperatively, and another patient developed intractable bradycardia requiring placement of permanent pacemaker. Demographics, NYHA classification, baseline patient characteristics, number of vessels grafted and the clinical end points are presented in Table 1. 
Table 1. Demographics and Patients Characteristics

\begin{tabular}{|c|c|}
\hline \multicolumn{2}{|l|}{ Demographics } \\
\hline Age (years) & $64.32 \pm 8.445$ \\
\hline Body Weight (kg) & $73.27 \pm 12.05$ \\
\hline Height $(\mathrm{cm})$ & $167.49 \pm 6.83$ \\
\hline Body Mass Index $\left(\mathrm{kg} / \mathrm{m}^{2}\right)$ & $26.09 \pm 3.56$ \\
\hline Male Gender & $68.3 \%$ \\
\hline Female Gender & $31.7 \%$ \\
\hline \multicolumn{2}{|l|}{ NYHA Classification } \\
\hline Class I & $15.2 \%$ \\
\hline Class II & $73.8 \%$ \\
\hline Class III & $10.3 \%$ \\
\hline Class IV & $0.7 \%$ \\
\hline \multicolumn{2}{|l|}{ Baseline patients characteristics } \\
\hline $\begin{array}{l}\text { Preoperative Ejection } \\
\text { Fraction }(\%)\end{array}$ & $\begin{array}{l}57.75 \% \pm \\
7.16 \%\end{array}$ \\
\hline Preoperative LVEDD(mm) & $52.17 \pm 5.82$ \\
\hline Hypertension & $24.1 \%$ \\
\hline Diabetes Mellitus & $74.5 \%$ \\
\hline $\begin{array}{l}\text { Previous Myocardial } \\
\text { Infarction }\end{array}$ & $20 \%$ \\
\hline Old Stroke or TIA & $15.86 \%$ \\
\hline Previous PCI & $11.72 \%$ \\
\hline \multicolumn{2}{|l|}{ Number of Vessels grafted } \\
\hline Single graft & $5(3.4 \%)$ \\
\hline Double grafts & $29(20 \%)$ \\
\hline Triple grafts & $81(55.9 \%)$ \\
\hline Four grafts & $28(19.3 \%)$ \\
\hline Five grafts & $2(1.4 \%)$ \\
\hline \multicolumn{2}{|l|}{ Clinical End Points (number of patients) } \\
\hline ICU $>5$ days postoperatively & 17 \\
\hline $\begin{array}{l}\text { Ventilator }>2 \text { days } \\
\text { postoperatively }\end{array}$ & 14 \\
\hline $\begin{array}{l}\text { Postoperative days in hospital } \\
>10 \text { days }\end{array}$ & 39 \\
\hline
\end{tabular}

$\mathrm{ICU}=$ Intensive care unit

NYHA $=$ New York heart association

LVEDD $=$ Left ventricular end diastolic diameter.

$\mathrm{TIA}=$ Transient ischemic attack

$\mathrm{PCI}=$ Percutaneous coronary intervention
Our results showed positive correlation of preoperative BNP levels with age and left ventricular end diastolic diameter (LVEDD). There was significant negative correlation with left ventricular ejection fraction (LVEF). There was no difference between the preoperative BNP and gender or the Basal Metabolic Index (BMI) of the patient. Associations between the preoperative BNP levels and other variables are shown in Table 2.

Table 2. Correlation with Preoperative BNP levels

\begin{tabular}{|l|c|c|}
\hline $\begin{array}{c}\text { Preoperative BNP levels } \\
\text { Correlation }\end{array}$ & Pearsons R & P-Value \\
\hline $\begin{array}{l}\text { Preoperative Echocardi- } \\
\text { ography Ejection Fraction }\end{array}$ & -0.379 & $<0.01$ \\
\hline $\begin{array}{l}\text { Preoperative Echocardi- } \\
\text { ography Left Ventricular } \\
\text { End Diastolic Diame- } \\
\text { ter(mm) }\end{array}$ & 0.200 & $<0.05$ \\
\hline $\begin{array}{l}\text { Age } \\
\left.\text { Body Mass Index (kg/m }{ }^{2}\right)\end{array}$ & -0.55 & \\
\hline $\begin{array}{l}\text { Number of Vessels } \\
\text { Grafted }\end{array}$ & -0.138 & NS \\
\hline $\begin{array}{l}\text { Preoperative Cardiac } \\
\text { hospital }\end{array}$ & 0.044 & NS \\
\hline
\end{tabular}

$\mathrm{NS}=$ Non significant

Statistically significant results were found when preoperative BNP was compared with baseline patient character. The plasma levels of preoperative BNP provide a better estimate of LVEF. We found that patients whose LVEF was 0.50 or less had higher BNP plasma concentrations. And the preoperative NYHA also had a statistically significant correlation with preoperative BNP. But the preoperative BNP concentration was not significantly different among the patients who had and who did not have previous myocardial infarction (Table 3). 
Table 3. Log transformed preoperative BNP levels and Patient Characteristics

\begin{tabular}{|l|c|c|c|}
\hline & $\begin{array}{c}\text { Number } \\
\text { of } \\
\text { Patients }\end{array}$ & $\begin{array}{c}\text { Mean of log } \\
\text { transformed } \\
\text { BNP-1 } \pm \text { SD }\end{array}$ & P value \\
\hline MI(no) & 116 & $1.68 \pm 0.48$ & 0.33 \\
\hline MI(yes) & 29 & $1.78 \pm 0.53$ & \\
\hline LVEF $\leq 50 \%$ & 25 & $2.09 \pm 0.48$ & \multirow{2}{*}{0.00083} \\
\hline LVEF $\geq 50 \%$ & 120 & $1.62 \pm 0.45$ & \\
\hline NYHA (I, II) & 129 & $1.67 \pm 0.48$ & 0.02 \\
\hline NYHA (III,IV) & 16 & $1.97 \pm 0.48$ & \\
\hline
\end{tabular}

$\mathrm{MI}=$ Myocardial Infarction

$\mathrm{EF}=$ Ejection Fraction

NYHA $=$ New York Heart Association

Preoperative BNP levels ranged from 5.2 to $796 \mathrm{pg} /$ $\mathrm{ml}$. Hence the groups of preoperative BNP level were dichotonized to being less than $100 \mathrm{pg} / \mathrm{ml}$ and greater than $100 \mathrm{pg} / \mathrm{ml}$. On serial follow-up, BNP levels increased over time after completion of the operation in both the groups. The change in BNP over time is depicted in Figure 1.

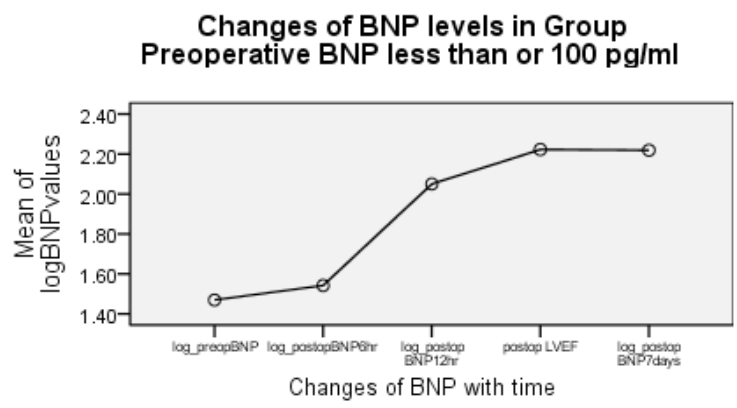

Changes of BNP levels in Group Preoperative BNP more than $100 \mathrm{na} / \mathrm{ml}$

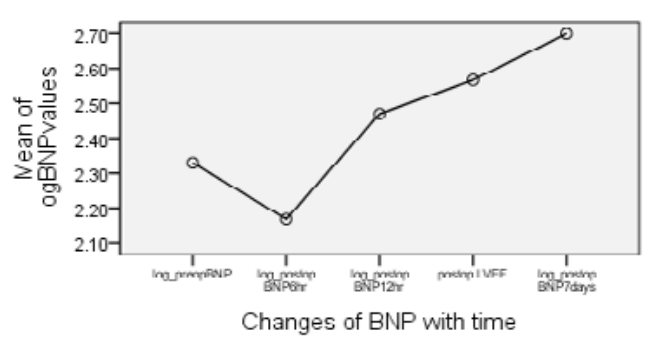

Figure 1: The changes in BNP levels over time

12 hour postoperative BNP level proved to be a valuable asset in this research as it had an important correlation with the duration of mechanical ventilation, and the postoperative length of hospital stay. The relationship between both the log-transformed hours of mechanical ventilation and the $\log$ transformed postoperative days of hospital stay and BNP levels were linear (Figure 2).

\section{Normal P-P Plot of Regression Standardized Residual of 12 hour BNP level vs Postoperative length of hospital stay}

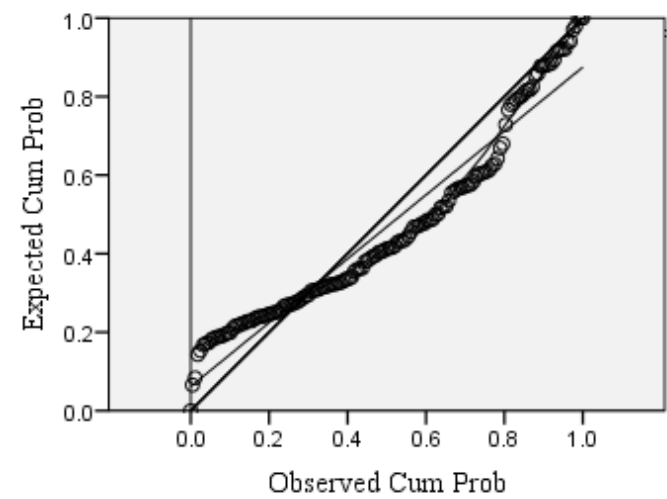

Normal P-P Plot of Regression Standardized Residual of 12

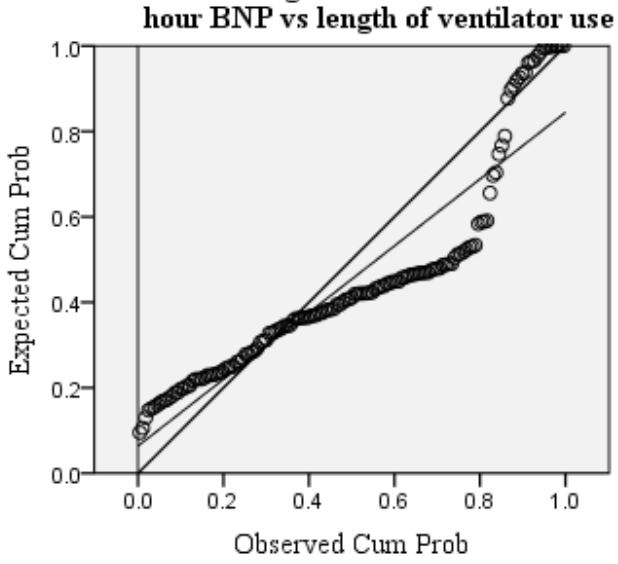

Figure 2: Normal P-P Plot of Regression Standardized Residual of 12 hour postoperative BNP level vs. Postoperative Hospital Stay and length of ventilator use

Next, the logistic regression model was fitted to find the association between the difference of the 12 hour postoperative BNP levels and the clinical outcome. The model was adjusted for potential confounders including age, weight and preexisting hypertension. After adjustment, a significant association was observed between 12 hour postoperative BNP level and the requirement of mechanical ventilation.

A receiver operating curve (ROC), which was used to evaluate the various cutoff levels of 12 hour postoperative BNP level to predict the need for mechanical ventilation beyond 48 hours, was derived as shown in Figure 3. Two 
computer-generated BNP cut-points were examined to calculate sensitivity and specificity. Prediction of the postoperative requirement of ventilator beyond 48 hours for higher BNP cut-point of $234 \mathrm{pg} / \mathrm{ml}$ had a sensitivity of $50 \%$ and a specificity of $89 \%$ and the lower BNP cut-point of 142 $\mathrm{pg} / \mathrm{ml}$ was more sensitive, with a sensitivity of $71.4 \%$, but less specific, with a specificity of 52.7. The area under the curve (AUC) was 0.646 .

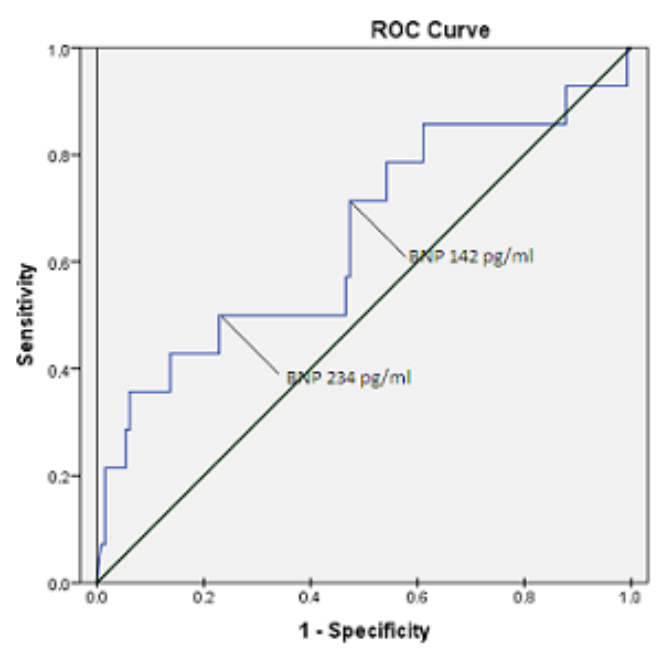

Figure 3 : ROC Curve 12 hour. postoperative BNP levels vs. Requirement of Ventilator of beyond 48 hours

\section{DISCUSSION}

Since the discovery of BNP, and, its diagnostic and prognostic properties in patients with left ventricular dysfunction, considerable interest have been generated for different practical uses of this cardiac neurohormone. ${ }^{3,9,10}$ In this study, we have assessed whether BNP measurement carried out before and after OPCAB surgery could provide information regarding the outcome of the operation.

Various patient characteristics including gender, age and BMI and were assessed separately to find a correlation with the levels of BNP. We found a positive correlation between preoperative BNP levels with age of the patient. BNP concentrations increase with age, as the left ventricle appears to stiffen over time, offering a likely stimulus to BNP production. ${ }^{7}$ In a meta-analyses study, Balion et al. ${ }^{10}$ described that among the demographic determinants, age was the most frequently reported determinant positively correlated with BNP. In the present study, however, we couldn't yield any strong association of BNP with gender, or the BMI of the patient.
Another important and novel finding of this study is that we found plasma BNP concentration was a predictor of cardiac function in patients undergoing OPCAB grafting. Preoperative BNP concentrations were much higher in patients with LVEF less than 50\%, owing to the low cardiac output state of the patients. Osman et al. ${ }^{6}$ reported that patients whose LVEF was $40 \%$ or less had higher BNP plasma concentrations. We report that the preoperative NYHA also had a statistically significant correlation with preoperative BNP which justify that patients with symptomatic left ventricular systolic dysfunction have increased BNP levels that correlate with NYHA classification.

As BNP is rapidly released by myocytes and disappears rapidly from plasma because of its short half-life of 22 minutes, ${ }^{11}$ we investigated its use in patients undergoing OPCAB surgery. We observed that the BNP levels rapidly elevated after the operation and remained higher than the preoperative level in our patient population. It is postulated that the increment of BNP levels in CABG patients may be due to the augmented regional wall stretch induced by perioperative myocardial ischemia in a non specific way. ${ }^{11}$, 12, 13 Such factors may include operative stress like general anesthesia, cardiac herniation, stabilizer compression, regional blood flow blockage, and reperfusion injury. ${ }^{13}$

We could not establish postoperative BNP values measured at 6 hour, 24 hour, and 7 days as significant predictors of clinical outcome. Nevertheless, we still uncovered some undeviating results of the potential capacity of the 12 hour postoperative BNP level to independently predict operative outcome in the course of OPCAB surgery. After adjustment of several confounders, notably age, weight and pre-existing hypertension, the results showed that patients having higher 12 hour postoperative BNP level required ventilator beyond 48 hour. This indicated 12 hour postoperative BNP level was an independent predictor of the duration of mechanical ventilation and the presence of a low cardiac output state within the first 48 hour postoperatively. Beside, the patients who had higher level of 12 hour postoperative BNP level also had a longer hospital stay. Few studies have shown similar findings, where they have stated that the patients with higher BNP level required significantly longer ventilation, increased length of ICU stay and longer hospital stay. ${ }^{5,7,14,15}$

The duration of mechanical ventilation is commonly interpreted as an important marker of disease severity. ${ }^{15}$ Higher BNP level had an association with the longer 
duration of weaning which can increase the complications arising from ventilator dependency. ${ }^{8}$ Our study showed that lower 12 hour postoperative BNP levels, tested by a single inexpensivepointofcareassay, canexcludesignificantcardiac dysfunction, thereby shortening the weaning period. On the other hand, our results also entails that it is prudent to more closely monitor patients with higher 12 hour postoperative BNP levels for possible postoperative complications.

ROC curve revealed 12 hour postoperative BNP level of higher cut-point of $234 \mathrm{pg} / \mathrm{ml}$ had a sensitivity of $50 \%$, a specificity of $89 \%$, and the lower BNP cut-point of $142 \mathrm{pg} /$ $\mathrm{ml}$ had a sensitivity of $71.4 \%$, and a specificity of $52.7 \%$. It should be noted that BNP, despite high specificity and accuracy, had low sensitivity and positive predictive value in our study. The AUC for requirement of ventilator beyond 48 hour was 0.65 . But having only 14 positive subjects with longer weaning period in our study is the most likely explanation for obtaining this satisfactory AUC value. Nonetheless, this report seems very promising for future studies with more statistical power. At this conjecture, it can be suggested that BNP levels are best used not as a "standalone" test, but in conjunction with existing multivariable risk indexes.
A few limitations of the present study are noteworthy. Our study is lacking the results of the long-term followup. Aside from heart condition, BNP levels may also rise in pulmonary embolism, pulmonary hypertension, renal failure, cirrhosis, and conditions common in intensive care units. ${ }^{16}$ More importantly, in this study we did not document all postoperative predictive values of BNP, for example, incidence of postoperative atrial fibrillation, postoperative infection, and re-hospitalization. A more definite conclusion about assay specific perioperative BNP cutoffs for risk prediction and estimation of prognosis after OPCAB surgery will require further studies.

\section{CONCLUSION}

This study suggests that plasma BNP concentration can be beneficial in predicting the risk and operative outcome in patients undergoing OPCAB grafting. We strongly believe that BNP is a valuable, inexpensive, reliable, simple and strong predictor of the outcome post-cardiac surgery and should be measured alongside the routine blood tests. We also suggest that it is incisive to more carefully monitor and cautiously manage patients with higher BNP levels for possible postoperative complications after OPCAB.

\section{REFERENCES}

1. Hua Liu, Chunsheng Wang, Lan Liu, et al. Perioperative application of $\mathrm{N}$-terminal pro-brain natriuretic peptide in patients undergoing cardiac surgery. Journal of Cardiothoracic Surgery; 2013;8:1

2. James A. De Lemos, David A. Morrow, Jane H. Bentley, et al. The prognostic value of B-type natriuretic peptide in patients with acute coronary syndromes. N Engl J Med; 2001; 345(14):10141021

3. Saina Attaran, Roy Sherwood, Jatin Desai, et al. Brain natriuretic peptide a predictive marker in cardiac surgery. Interactive CardioVascular and Thoracic Surgery; 2009;9:662-666

4. Oussama M. Wazni, David O. Martin, Nassir F. Marrouche, et al. Plasma B-Type Natriuretic Peptide Levels Predict Postoperative Atrial Fibrillation in Patients Undergoing Cardiac Surgery. Circulation; 2004;110:124-127

5. Zanxin Wang, Degang Liang, Qiang Fu, et al. Perioperative brain natriuretic peptide in offpump coronary artery bypass; ActaCardiol; 2010;65(3):297-301

6. Osman Saribulbul, Ilker Alat, Senol Cosku, et.al. The Role of Brain Natriuretic Peptide in the Prediction of Cardiac Performance in Coronary Artery Bypass Grafting. Tex Heart Inst J ; 2003;30:298-304

7. Łukasz J. Krzych, Dariusz Szurlej, Tadeusz Kołodziej, et al. Diagnostic accuracy of preoperative NT-proBNP level in predicting shortterm outcomes in coronary surgery: a pilot study. Kardiologia Polska; 2011;69(11):1121-1127

8. Mekontso Dessap, L. Brochard. B-type natriuretic peptide for the management of weaning (BMW study). Protocol presentation, Réanimation; 2008; 17:57-65 
9. Jenny Doust, Richard Lehman, Paul Glasziou, The Role of BNP Testing in Heart Failure, American Family Physician; 2006;74(11):1893-1898.

10. Cynthia Balion, Parminder Raina, et al. Testing for BNP and NT-proBNP in the Diagnosis and Prognosis of Heart Failure, AHRQ; 2006;142:1-147

11. Georges, F. Forestier, N. Valli, et al. Changes in type B natriuretic peptide (BNP) concentrations during cardiac valve replacement, European Journal of Cardio-thoracic Surgery; 2004;25:941945

12. Konstantinos Tziomalos, Julia Sevastidou, Antonios A. Pitsis, et.al. The Potential Predictive Value of Preoperative Brain Natriuretic Peptide Levels in Coronary Artery Bypass Grafting Surgery, The Open Cardiovascular and Thoracic Surgery Journal; 2008;1:27-36

13. Min-Ho Song, Yoshie Kobayashi, Hiroyasu Michi. Natriuretic Peptide in Coronary Artery Bypass Grafting, Asian CardiovascThorac Ann; 2004;12:41-46
14. Chie-Youn Shih, Anil Sapru, Peter Oishi, et.al. Alterations in plasma B-type natriuretic peptide levels after repair of congenital heart defects: A potential perioperative marker, J ThoracCardiovascSurg; 2006;131:632-638

15. Thomas Schachner, Dominik Wiedemann, Hannes Fetz, et al. Influence of preoperative serum $\mathrm{N}$-terminal pro-brain type natriuretic peptide on the postoperative outcome and survival rates of coronary artery bypass patients, Clinics; 2010;65(12):1239-1245

16. Roberto Bassan, Alfredo Potsch, Alan Maisel et al. B-type natriuretic peptide: a novel early blood marker of acute myocardial infarction in patients with chest pain and no ST-segment elevation. European Heart Journal; 2005;26:234-240 